\title{
RADIATION LOSSES IN MICROSTRIP ANTENNA FEED NETWORKS PRINTED ON MULTILAYER SUBSTRATES*
}

\author{
The Radiation Laboratory; Electrical Engineering and Computer Science Department, 1301 Beal Ave., University of \\ Michigan, Ann Arbor, MI 48109, U.S.A.
}

\begin{abstract}
SUMMARY
The effect of the substrate structure on the radiation properties of microstrip array feed networks is investigated with the space-domain integral equation approach. Numerical and analytical techniques are employed to produce efficient computer algorithms. Results for space and surface wave losses are presented for corner discontinuities printed on substrate/superstrate, and two-layer substrate structures. Comparisons are made with the single-layer case.
\end{abstract}

\section{INTRODUCTION}

Planar integration of active devices with passive radiating elements offers many advantages such as reduced area and shorter interconnect lines. Unfortunately, such integration schemes suffer for a variety of reasons, including the availability of reliable models for passive circuits and radiating elements. In most monolithic array applications, the feeding structure and antenna elements are made of microstrip. Despite the advantages of the microstrip technology, radiation from discontinuities included in microstrip feed structures ${ }^{1-7}$ reduces the gain of the antenna, and deteriorates the array patterns. As a result, models for this loss mechanism on the array performance should become an important part of the array design procedure.

Microstrip arrays often have, for their protection, a cover or superstrate layer, which has also been reported to improve the gain of microstrip dipoles. This gain-enhancement technique, based on the elimination of surface waves, has been discussed extensively in the literature. ${ }^{8.9}$ As mentioned in this work, total elimination of the surface waves is not practicable with commercially available substrates; however, a moderate improvement in gain is realizable. Nonetheless, the presence of the superstrate, whether it is used in a planar array for improved performance or protection, must be considered carefully. In this planar configuration, the superstrate increases the substrate thickness, which may trigger higher radiation in the feed network. These higher losses can offset the increase in array gain and further complicate the design. Furthermore, superstrates reduce the operating frequency range by lowering the cut-off frequencies of the higher-order microstrip and surface wave modes.

In this paper, a full-wave method of moments technique ${ }^{7}$ is employed to analyse the radiation properties of microstrip discontinuities often encountered in feeding networks printed on multilayer substrates. The space and surface wave contributions ${ }^{10-13}$ are evaluated and it is demonstrated that the utilization of a superstrate may result in higher radiation losses and lower overall array gain.

\section{THEORY}

Consider the open microstrip structure having a multilayer substrate as shown in Figure 1(a) or a superstrate shown in Figure 1(b). The conductors are lossless and their thickness $(t)$ is much smaller than a wavelength. The substrate is of thickness $h$, and is also assumed lossless. The electric field may be written in terms of the electric current density as shown below

"Invited Paper. 

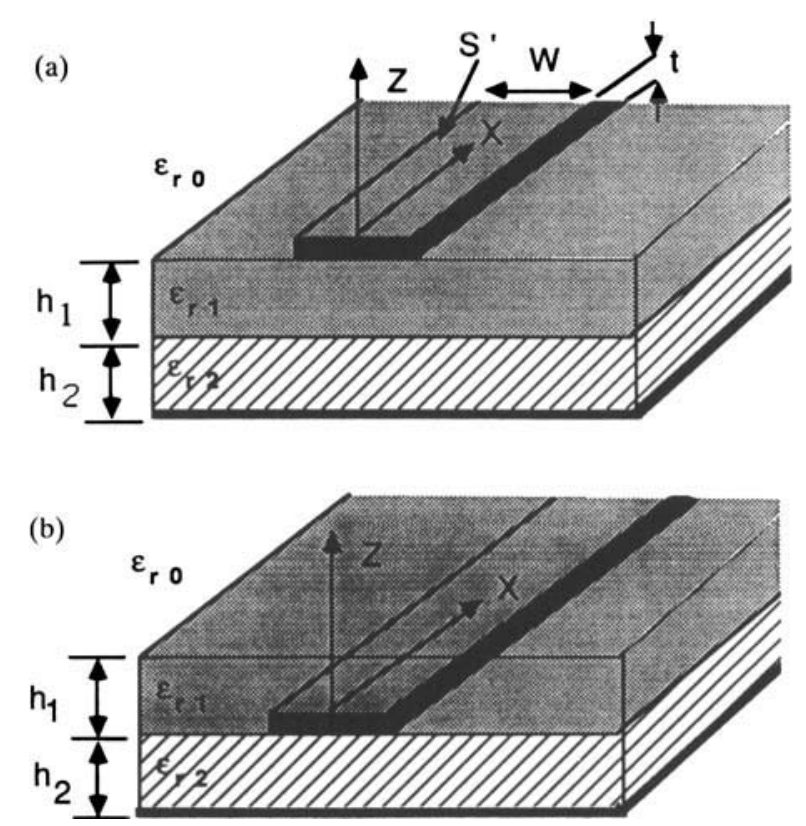

Figure 1. Multilayer open microstrip geometry with and without a superstrate. (a) Microstrip discontinuity with multilayer substrate. (b) Microstrip discontinuity with superstrate

$$
\mathbf{E}(x, y, z)=\left.\iint_{s^{\prime}}\left[k_{i}^{2} l+\nabla \nabla\right] \cdot \mathbf{G}_{i}\left(x, y, z / x^{\prime}, y^{\prime}, z^{\prime}\right) \cdot \mathbf{J}\left(x^{\prime}, y^{\prime}\right)\right|_{z^{\prime}=0} \mathrm{~d} s^{\prime} \quad(i=0,1,2)
$$

where $k_{i}$ and $\mathbf{G}_{i}\left(x, y, z / x^{\prime}, y^{\prime}, z^{\prime}\right)$ are the wavenumber and dyadic Green's function in region (i), and

$$
\mathrm{J}\left(x^{\prime}, y^{\prime}\right)=J_{x}\left(x^{\prime}, y^{\prime}\right) \hat{x}+J_{y}\left(x^{\prime}, y^{\prime}\right) \hat{y}
$$

is the planar current density on the conducting strips. The components of the dyadic Green's function used in equation (1) are expressed in terms of Sommerfeld integrals ${ }^{7,14}$ as shown below

$$
\begin{aligned}
G_{\xi \xi}\left(x, y, z / x^{\prime}, y^{\prime}, 0\right) & =\left(\frac{\omega \mu_{0}}{2 \pi k_{0}^{2}}\right) \int_{0}^{\infty} J_{0}(\lambda \rho) \mathscr{Z}_{\xi \xi}(z) \frac{\mathcal{N}_{\xi \xi}(\lambda)}{f_{1}(\lambda)} \mathrm{d} \lambda \\
G_{z \xi}\left(x, y, z / x^{\prime}, y^{\prime}, 0\right) & =\left(\frac{\omega \mu_{0}}{2 \pi k_{0}^{2}}\right) \Phi(\phi) \int_{0}^{\infty} J_{1}(\lambda \rho) \mathscr{Z}_{z \xi}(z) \frac{\mathcal{N}_{z \xi}(\lambda)}{f_{1}(\lambda) f_{2}(\lambda)} \mathrm{d} \lambda \\
\xi & =x, y
\end{aligned}
$$

where

$$
\begin{aligned}
\rho & =\sqrt{\left(x-x^{\prime}\right)^{2}+\left(y-y^{\prime}\right)^{2}} \\
\Phi(\phi) & = \begin{cases}\cos (\phi) & \xi=x \\
\sin (\phi) & \xi=y\end{cases}
\end{aligned}
$$

and with $\mathcal{N}_{\xi \xi}(\lambda), \mathcal{N}_{z \xi}(\lambda), f_{1}(\lambda)$, and $f_{2}(\lambda)$ given in Appendix A. In equations (3) and (4), $f_{1}(\lambda)$, and $f_{2}(\lambda)$ are analytic functions with discrete zeros. The contributions from these zeros give the power propagating in the substrate in the form of transverse electric (TE) and transverse magnetic (TM) surface waves, respectively.

To obtain the electric current density over the conducting strips, the method of moments is applied. ${ }^{15} \mathrm{~A}$ rectangular region containing the microstrip discontinuity is subdivided into rectangles (see Figure 2) and the current is expressed as a superposition of known rooftop basis functions ${ }^{16}$ multiplied by unknown coefficients. 


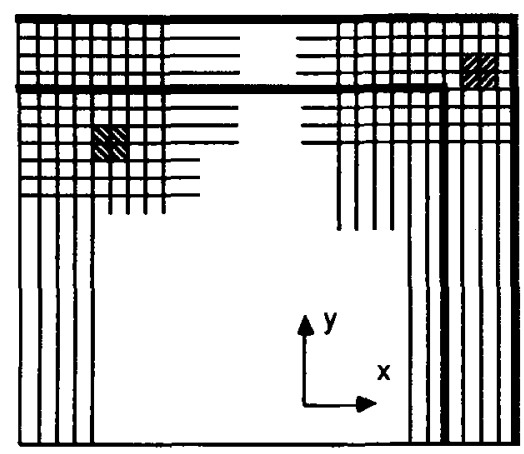

(a)

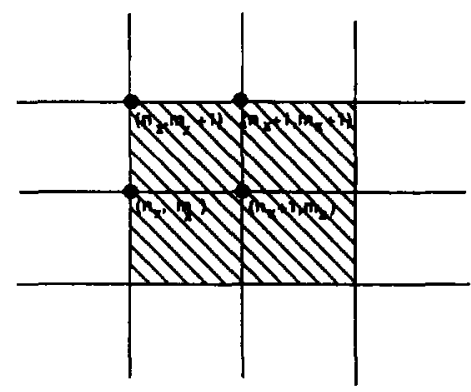

(b)

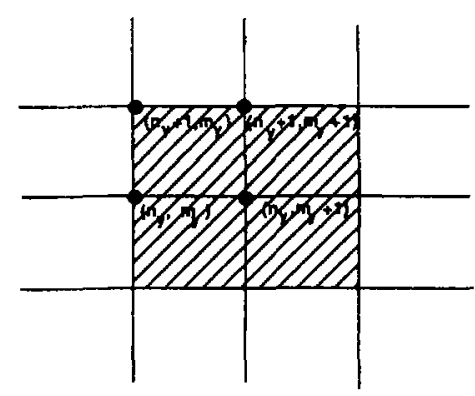

(c)

Figure 2. Subdivision of (M)MIC area around corner discontinuity. (a) Typical discretization; (b) $x$-directed mesh; (c) $y$ directed mesh

$$
\begin{aligned}
& J_{x}\left(x^{\prime}, y^{\prime}\right)=\sum_{n_{x}=1}^{N_{x}+1} \sum_{m_{x}=1}^{M_{x}+1} I_{n m}^{x}\left[f_{n_{x}}\left(x^{\prime}\right) g_{m_{x}}\left(y^{\prime}\right)\right] \\
& J_{y}\left(x^{\prime}, y^{\prime}\right)=\sum_{n_{y}=1}^{N_{y}+1} \sum_{m_{y}=1}^{M_{y}+1} I I_{n m}^{y}\left[f_{n_{y}}\left(y^{\prime}\right) g_{m_{y}}\left(x^{\prime}\right)\right]
\end{aligned}
$$

where the pairs $\left(n_{x}, m_{x}\right)$ and $\left(n_{y}, m_{y}\right)$ indicate the nodes in the mesh for the $x$ - and $y$-current, respectively. In addition, the function $f_{n_{\xi}}\left(\xi^{\prime}\right)$ gives the longitudinal dependence of each component

$$
f_{n_{\xi}}\left(\xi^{\prime}\right)=\left\{\begin{array}{cc}
\frac{\sin k_{s}\left(\xi_{n_{\xi}+1}-\xi^{\prime}\right)}{\sin k_{s} l_{\xi}} & \xi_{n_{\xi}} \leqslant \xi^{\prime} \leqslant \xi_{n_{\xi}+1} \\
\frac{\sin k_{s}\left(\xi^{\prime}-\xi_{n_{\xi}-1}\right)}{\sin k_{s} l_{\xi}} & \xi_{n_{\xi}-1} \leqslant \xi^{\prime} \leqslant \xi_{n_{\xi}} \\
0 & \text { else } \\
& (\xi, \zeta)=(x, y),(y, x)
\end{array}\right.
$$

while $g_{m_{\xi}}\left(\zeta^{\prime}\right)$ gives the transverse dependence

$$
g_{m_{\xi}}\left(\zeta^{\prime}\right)=\left\{\begin{aligned}
1 & \zeta_{m_{\xi}} \leqslant \zeta^{\prime} \leqslant \zeta_{m_{\xi}+1} \\
0 & \text { else } \\
& (\xi, \zeta)=(x, y),(y, x)
\end{aligned}\right.
$$

In the above, $l_{\xi}=\xi_{n_{\xi}+1}-\xi_{n_{\xi}}$, and $k_{s}$ is a scaling parameter chosen to vary between $k_{0}$ (free space wavenumber) and $k_{i}$ (wavenumber in the highest permittivity dielectric region).

With the substitution of equations (7)-(10) into equation (1), the original integral equation can be written in the form 


$$
\begin{aligned}
E_{x}+\Delta E_{z} & =\sum_{n_{x}=1}^{N_{x}+1} \sum_{m_{x}=1}^{M_{x}+1} I_{n m}^{x} \iint_{S^{\prime}} \mathscr{K}_{x x}\left(x, y / x^{\prime}, y^{\prime}\right) f_{n_{x}}\left(x^{\prime}\right) g_{m_{x}}\left(y^{\prime}\right) \mathrm{d} x^{\prime} \mathrm{d} y^{\prime} \\
& +\sum_{n_{y}=1}^{N_{y}+1} \sum_{m_{y}=1}^{M_{y}+1} I_{n m}^{y} \iint_{S^{\prime}} \mathscr{K}_{x y}\left(x, y / x^{\prime}, y^{\prime}\right) f_{n_{y}}\left(y^{\prime}\right) g_{m_{y}}\left(x^{\prime}\right) \mathrm{d} x^{\prime} \mathrm{d} y^{\prime} \\
E_{y}+\Delta E_{y} & =\sum_{n_{x}=1}^{N_{x}+1} \sum_{m_{x}=1}^{M_{x}+1} I_{n m}^{x} \iint_{S^{\prime}} \mathscr{K}_{y x}\left(x, y / x^{\prime}, y^{\prime}\right) f_{n_{x}}\left(x^{\prime}\right) g_{m_{x}}\left(y^{\prime}\right) \mathrm{d} x^{\prime} \mathrm{d} y^{\prime} \\
& +\sum_{n_{y}=1}^{N_{y}+1} \sum_{m_{y}=1}^{M_{y}+1} I_{n m}^{y} \iint_{S^{\prime}} \mathscr{K}_{y y}\left(x, y / x^{\prime}, y^{\prime}\right) f_{n_{y}}\left(y^{\prime}\right) g_{m_{y}}\left(x^{\prime}\right) \mathrm{d} x^{\prime} \mathrm{d} y^{\prime}
\end{aligned}
$$

where $\mathscr{K}_{\xi \zeta}\left(x, y / x^{\prime}, y^{\prime}\right)(\xi, \zeta=x, y)$ are integro-differential operators given by

$$
\mathscr{K}_{\xi \zeta}\left(x, y / x^{\prime}, y^{\prime}\right)=\int_{0}^{\infty}\left[\left(k_{i}^{2} \delta_{\xi \zeta}+\frac{\partial^{2}}{\partial \xi \partial \zeta}\right) F_{\zeta \zeta}+\frac{\partial^{2}}{\partial z \partial \xi} F_{z \zeta}\right] \mathrm{d} \lambda
$$

$\delta_{\xi \zeta}$ is the Kronecker delta, and $F_{\zeta \zeta}$ and $F_{z \zeta}$ are functions of $\lambda$ of the following form

$$
\begin{aligned}
& F_{\zeta \zeta}=\left(\frac{\omega \mu_{0}}{2 \pi k_{0}^{2}}\right) J_{0}(\lambda \rho) \mathscr{E}_{\zeta \zeta}(z) \frac{\mathcal{N}_{\zeta \zeta}(\lambda)}{f_{1}(\lambda)} \\
& F_{z \zeta}=\left(\frac{\omega \mu_{0}}{2 \pi k_{0}^{2}}\right) \Phi(\phi) J_{1}(\lambda \rho) \mathscr{E}_{z \zeta}(z) \frac{N_{z \zeta}(\lambda)}{f_{1}(\lambda) f_{2}(\lambda)}
\end{aligned}
$$

In equations (11) and (12) the errors $\Delta E_{x}$ and $\Delta E_{y}$ are introduced under the approximations made for the unknown current distributions in equations (7)-(10). The $z$ derivative in equation (13) may be replaced by an $\zeta$ derivative resulting in the modified form for the operator $\mathscr{K}_{\xi \zeta}$ :

$$
\begin{aligned}
& \mathcal{X}_{\xi \zeta}\left(x, y / x^{\prime}, y^{\prime}\right)=\int_{0}^{\infty}\left[\left(k_{i}^{2} \delta_{\xi \zeta}+\frac{\partial^{2}}{\partial \xi \partial \zeta}\right) f_{\zeta \zeta}+\frac{\partial^{2}}{\partial \xi \partial \zeta} f_{z \zeta}\right] J_{0}(\lambda \rho) \mathrm{d} \lambda \\
& f_{\zeta \zeta}=F_{\zeta \zeta} \\
& f_{z \zeta}=-\left(\frac{\omega \mu_{0}}{2 \pi k_{0}^{2}}\right) \mathscr{F}^{\prime}{ }_{z \zeta}(z) \frac{\mathcal{N}_{z \zeta}(\lambda)}{\lambda f_{1}(\lambda) f_{2}(\lambda)}
\end{aligned}
$$

where $\mathscr{Z}_{z \zeta}^{\prime}(z)$ is the first derivative of $\mathscr{Z}_{z \zeta}(z)$ with respect to $z$. In this manner, the first-order Bessel function in equation (15) is eliminated, and the $\rho$-dependence in all Sommerfeld integrals is in the argument of a zeroth-order Bessel function of the first kind.

The application of Galerkin's method reduces equations (11) and (12) to a matrix equation

$$
[\mathbf{Z}][\mathbf{I}]=[\mathbf{V}]
$$

where $\mathbf{Z}$ is the impedance matrix, $\mathbf{I}$ is the vector of unknown $x$ and $y$ current amplitudes, and $\mathbf{V}$ is the excitation vector. The impedance matrix is a square matrix containing four submatrices as shown below

$$
[\mathbf{Z}]=\left[\begin{array}{ll}
{\left[Z_{x x}\right]} & {\left[Z_{x y}\right]} \\
{\left[Z_{y x}\right]} & {\left[Z_{y y}\right]}
\end{array}\right]
$$

The elements of each submatrix are given by

$$
\begin{aligned}
& Z_{x x}(n, m, \nu, \mu)=\left\langle f_{n_{x}}\left(x^{\prime}\right) g_{m_{x}}\left(y^{\prime}\right), \mathscr{K}_{x x}, f_{\nu_{x}}(x) g_{\mu_{x}}(y)\right\rangle \\
& Z_{x y}(n, m, \nu, \mu)=\left\langle f_{n_{x}}\left(x^{\prime}\right) g_{m_{x}}\left(y^{\prime}\right), \mathscr{K}_{x y}, f_{v_{y}}(y) g_{\mu_{y}}(x)\right\rangle
\end{aligned}
$$




$$
\begin{aligned}
& Z_{y x}(n, m, \nu, \mu)=\left\langle f_{n_{y}}\left(y^{\prime}\right) g_{m_{y}}\left(x^{\prime}\right), \mathscr{H}_{y x}, f_{\nu_{x}}(x) g_{\mu_{x}}(y)\right\rangle \\
& Z_{y y}(n, m, \nu, \mu)=\left\langle f_{n_{y}}\left(y^{\prime}\right) g_{m_{y}}\left(x^{\prime}\right), \mathscr{K}_{y y}, f_{\nu_{y}}(y) g_{\mu_{y}}(x)\right\rangle
\end{aligned}
$$

where the pair $\left(v_{\xi}, \mu_{\xi}\right)(\xi=x, y)$ indicates the testing points. The terms $Z_{x x}$ and $Z_{y y}$ are called the direct-coupled terms because the direction of the testing field and the current component are the same, while the terms $Z_{x y}$ and $Z_{y x}$ are the cross-coupled terms.

The double inner product in equations (21)-(24) is of the form

$$
\left\langle f_{n_{\xi}} g_{m_{\xi}}, \mathscr{K}_{\xi \zeta}, f_{v_{\zeta}} g_{\mu_{\zeta}}\right\rangle=\iint_{S^{\prime}} \mathrm{d} x^{\prime} \mathrm{d} y^{\prime} \iint_{S} \mathrm{~d} x \mathrm{~d} y\left(f_{n_{\xi}} g_{m_{\xi}} \mathscr{K}_{\xi \zeta} f_{\nu_{\zeta}} g_{\mu_{\zeta}}\right)
$$

where $S$, and $S^{\prime}$ represent the surface of the conducting strips.

\subsection{Evaluation of the impedance matrix}

The computation of the elements in the impedance matrix requires the evaluation of quadruple spatial integrals present in equation (25), as well as the semi-infinite Sommerfeld integrals in the Green's function. The Sommerfeld integral are computed by a real-axis integration in the complex $\lambda$-plane ${ }^{17}$ using an extraction of the singularities technique which effectively takes into account the contribution from the simple pole singularities of the integrand (see Figure 3 ). For a lossless substrate these poles lie on the real axis between the free space wavenumber $\left(k_{0}\right)$ and the highest wavenumber of the other layers $\left(\operatorname{MAX}\left(k_{1}, k_{2}\right)\right)$. The residues of the poles correspond to radiated power in the form of TM and TE modes propagating within the substrate layer. For the grounded substrate configuration, the $\mathrm{TM}_{0}$ surface wave mode has no cut-off frequency.

For simplicity in the computation, the semi-infinite Sommerfeld integrals are divided into two regions. A combination of numerical and analytical techniques is employed to evaluate the integrals in each region separately. The first region extends from 0 to the parameter $A$ and the second from $[A-\infty)$. The parameter $A$ is chosen to satisfy the condition

$$
A_{t}=\tanh \left(\sqrt{A^{2}-k_{i}^{2}} h_{i}\right) \doteq 1
$$

where the index (i) refers to the electrically thinnest dielectric layer which is adjacent to the microstrip structure. When $\lambda$ is greater than $A$, simplifications made in the integrand of the Sommerfeld integral result in improved accuracy and reduced numerical and computational effort.

In view of the above, the elements of the impedance matrix may be written as

$$
Z_{\xi \zeta}=Z_{\xi \zeta}^{A}+Z_{\xi \zeta}^{\infty} \quad \xi, \zeta=x, y
$$

2.1.1. Evaluation of $Z_{\xi \zeta}^{A}$. Considering equations (16), (25), and (26), $Z_{\xi \zeta}^{A}(n, m, \nu, \mu)$ is given by the following expression:

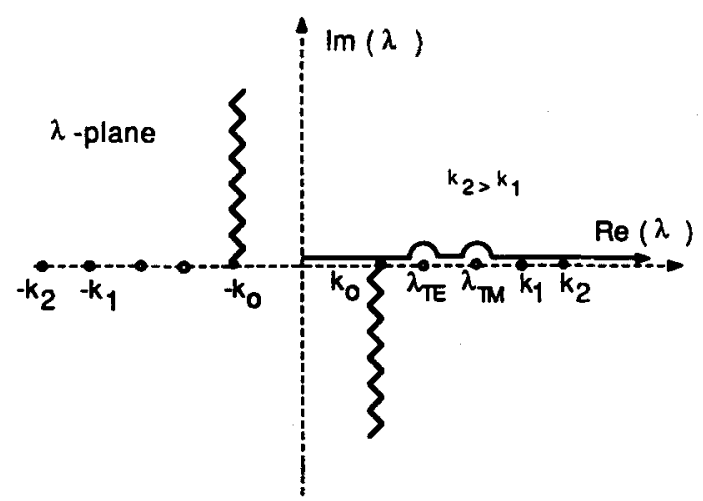

Figure 3. Real axis integration of Sommerfeld integral 


$$
\begin{aligned}
Z_{\xi \xi}^{A}(n, m, \nu, \mu) & =\delta_{\xi \zeta} \mathscr{L}_{\xi \xi}(A)\left\langle f_{n_{\xi}} g_{m_{\xi}}, J_{0}(\lambda \rho), f_{v_{\xi}} g_{\mu_{\xi}}\right\rangle \\
& +\mathscr{R}_{\xi \zeta}(A)\left\langle f_{n_{\xi}} g_{m_{\xi}}, \frac{\partial^{2}}{\partial \xi \partial \zeta} J_{0}(\lambda \rho), f_{\nu_{\xi}} g_{\mu_{\xi}}\right\rangle
\end{aligned}
$$

where $\mathscr{L}_{\xi \xi}(A)$ and $\mathscr{R}_{\xi \zeta}(A)$ are integral operators given by

$$
\begin{aligned}
& \mathscr{L}_{\xi \xi}(A)=k_{i}^{2} \int_{0}^{A} \mathrm{~d} \lambda f_{\xi \xi} \\
& \mathscr{R}_{\xi \zeta}(A)=\int_{0}^{A} \mathrm{~d} \lambda\left(f_{\zeta \zeta}+f_{z \zeta}\right)
\end{aligned}
$$

The real-axis evaluation of the Sommerfeld integrals with simple pole singularities is given in Reference 17. As mentioned, the double inner products contain quadruple integrals which would result in unacceptable numerical error and excessive CPU time, if the integrations were performed numerically. This difficulty has been overcome by reducing the integrals to convergent series. Along these lines, the Bessel function and its derivatives may be written in integral form as

$$
\begin{aligned}
J_{0}(\lambda \rho) & =\frac{1}{2 \pi} \int_{-\pi}^{\pi} \mathrm{e}^{j \lambda\left(x-x^{\prime}\right) \cos \phi} \mathrm{e}^{j \lambda\left(y-y^{\prime}\right) \sin \phi} \mathrm{d} \phi \\
\frac{\partial^{\sigma+\tau}}{\partial x^{\sigma} \partial y^{\tau}} J_{0}(\lambda \rho) & =\frac{1}{2 \pi} \int_{-\pi}^{\pi}(j \lambda \cos \phi)^{\sigma}(\mathrm{j} \lambda \sin \phi)^{\tau} \mathrm{e}^{j \lambda\left(x-x^{\prime}\right) \cos \phi} \mathrm{e}^{j \lambda\left(y-y^{\prime}\right) \sin \phi} \mathrm{d} \phi
\end{aligned}
$$

Employing these relations the quadruple integrals can be reduced to quickly converging series as shown in Appendix B. The series are of the form

$$
\begin{aligned}
& \left\langle f_{n_{\xi}} g_{m_{\xi}}, J_{0}(\lambda \rho), f_{v g_{\xi}} g_{\mu_{\xi}}\right\rangle \\
& =\sum_{k=0}^{\infty} \sum_{k^{\prime}=0}^{\infty} \sum_{l=0}^{\infty} \sum_{l^{\prime}=0}^{\infty} A_{2 k} A_{2 k^{\prime}} B_{l} B_{l^{\prime}}(-1)^{k+k^{\prime}}(-j)^{l+l^{\prime}} \frac{\partial^{2\left(k+k^{\prime}\right)+\left(l+l^{\prime}\right)} J_{0}(\lambda \rho)}{\left(\partial\left|\xi-\xi^{\prime}\right|\right)^{2\left(k+k^{\prime}\right)}\left(\partial\left|\zeta-\zeta^{\prime}\right|\right)^{\left(l+l^{\prime}\right)}} \\
& \left\langle f_{n_{\xi}} g_{m_{\xi}}, \frac{\partial^{2}}{\partial \xi^{2}} J_{0}(\lambda \rho), f_{v_{\xi}} g_{\mu_{\xi}}\right\rangle \\
& =\sum_{k=0}^{\infty} \sum_{k^{\prime}=0}^{\infty} \sum_{l=0}^{\infty} \sum_{l^{\prime}=0}^{\infty} A_{2 k} A_{2 k^{\prime}} B_{l} B_{l^{\prime}}(-1)^{k+k^{\prime}}(-j)^{l+l^{\prime}} \frac{\partial^{2\left(k+k^{\prime}+1\right)+\left(l+l^{\prime}\right)} J_{0}(\lambda \rho)}{\left(\partial\left|\xi-\xi^{\prime}\right|\right)^{2\left(k+k^{\prime}+1\right)}\left(\partial\left|\zeta-\zeta^{\prime}\right|\right)^{\left(l+l^{\prime}\right)}} \\
& \left\langle f_{n_{\xi}} g_{m_{\xi}}, \frac{\partial^{2}}{\partial \xi \partial \zeta} J_{0}(\lambda \rho), f_{v_{l}} g_{\mu_{\zeta}}\right\rangle \\
& =\sum_{k=0}^{\infty} \sum_{k^{\prime}=0}^{\infty} \sum_{l=0}^{\infty} \sum_{l^{\prime}=0}^{\infty} A_{2 k} A_{2 k^{\prime}} B_{l} B_{l^{\prime}}(-1)^{k+k^{\prime}}(-j)^{l+l^{\prime}} \frac{\partial^{2\left(k+k^{\prime}+1\right)+\left(l+l^{\prime}\right)} J_{0}(\lambda \rho)}{\left(\partial\left|\xi-\xi^{\prime}\right|\right)^{\left(2 k+l^{\prime}+1\right)}\left(\partial\left|\zeta-\zeta^{\prime}\right|\right)^{\left(2 k^{\prime}+l+1\right)}}
\end{aligned}
$$

where $\xi, \zeta=x, y(\xi \neq \zeta)$, and $\rho=\sqrt{\left(\xi_{\nu_{\xi}}-\xi_{n_{\xi}}\right)^{2}+\left(\zeta_{\mu_{\zeta}}-\zeta_{m_{\zeta}}\right)^{2}}$. These summations result in a substantial savings of CPU time over numerical integration.

2.1.2. Evaluation of $\boldsymbol{Z}_{\xi \zeta}^{\infty}$. The contribution for the interval from $(A, \infty)$ can be written

$$
\begin{aligned}
& Z_{x x}^{(x)}=\left\langle f_{n_{x}} g_{m_{x}}, \mathscr{K}_{x x}^{(x)}, f_{\nu_{x}} g_{\mu_{x}}\right\rangle \\
& Z_{x y}^{(x)}=\left\langle f_{n_{x}} g_{m_{x}}, \mathscr{K}_{x y}^{(x)}, f_{v_{y}} g_{\mu_{y}}\right\rangle \\
& Z_{y x}^{(x)}=\left\langle f_{n_{y}} g_{m_{y}}, \mathscr{K}_{y x}^{(x)}, f_{v_{x}} g_{\mu_{x}}\right\rangle \\
& Z_{y y}^{(x)}=\left\langle f_{n_{y}} g_{m_{y}}, \mathscr{K}_{y y}^{(x)}, f_{v_{y}} g_{\mu_{y}}\right\rangle
\end{aligned}
$$


where

$$
\mathscr{H}_{\xi \zeta}^{(\infty)}=\int_{A}^{\infty}\left[\left(k_{i}^{2} \delta_{\xi \zeta}+\frac{\partial^{2}}{\partial \xi \partial \zeta}\right) f_{\zeta \zeta}+\frac{\partial^{2}}{\partial \xi \partial \zeta} f_{z \zeta}\right] J_{0}(\lambda \rho) \mathrm{d} \lambda
$$

When $A$ is chosen according to equation (26), simplifications may be made in the integrand of the Green's function resulting in the expressions

$$
\mathscr{K}_{\xi \zeta}^{(x)}=\mathscr{H}_{\xi \zeta}^{(x)}-\mathscr{H}_{\xi \zeta}^{(A)}
$$

where

$$
\mathscr{H}_{\xi \zeta}^{(x)}=\left\{h_{\zeta \zeta}\left[\delta_{\xi \zeta}+\frac{\partial^{2}}{\partial \xi \partial \zeta}\right]+h_{z \zeta} \frac{\partial^{2}}{\partial \xi \partial \zeta}\right\} \frac{1}{\sqrt{\rho^{2}+\left(\frac{t}{f(A)}\right)^{2}}}
$$

and

$$
\mathscr{H}_{\xi \zeta}^{(A)}=\int_{0}^{A} \mathrm{~d} \lambda\left[h_{\zeta \zeta}\left(\delta_{\xi \zeta}+\frac{\partial^{2}}{\partial \xi \partial \zeta}\right)+h_{z \zeta} \frac{\partial^{2}}{\partial \xi \partial \zeta}\right] J_{0}(\lambda \rho) \frac{\mathrm{e}^{-\lambda f f(A)}}{f(A)}
$$

In equations (42) and (43), $f(A), h_{\zeta \zeta}$, and $h_{z \zeta}$ are constants given by

$$
\begin{aligned}
& h_{\zeta \zeta}=\left(\frac{\omega \mu_{0}}{2 \pi k_{0}^{2}}\right) \frac{1}{2\left[1-\epsilon_{2}(A)\right] f(A)} \\
& h_{z \zeta}=\left(\frac{\omega \mu_{0}}{2 \pi k_{0}^{2}}\right) \frac{1}{2 f(A)}\left(\frac{1}{\left[1-\epsilon_{2}(A)\right]}-\frac{2}{\left[\left(\epsilon_{r 2}+\epsilon_{r 1}\right)\left(1-\epsilon_{3}(A)\right)\right]}\right)
\end{aligned}
$$

with

$$
\begin{aligned}
f(A) & =\sqrt{1+\frac{k_{1}^{2}}{A^{2}-k_{1}^{2}}} \\
\epsilon_{2}(A) & =\frac{k_{2}^{2}-k_{1}^{2}}{4\left(A^{2}-k_{1}^{2}\right)} \\
\epsilon_{3}(A) & =\frac{\epsilon_{r 1}}{2\left(\epsilon_{r 1}+\epsilon_{r 2}\right)} \frac{k_{2}^{2}-k_{1}^{2}}{4\left(A^{2}-k_{1}^{2}\right)}
\end{aligned}
$$

for the case of a superstrate/substrate configuration. When the superstrate is not present $\epsilon_{r 1}$, and $\epsilon_{r 2}$ are replaced by the quantities $\epsilon_{r 1}$, and $\epsilon_{r 1}$, respectively.

Substitution of (41) into (36)-(39) gives

$$
Z_{\xi \zeta}^{(x)}=\left\langle f_{n_{\xi}} g_{m_{\xi}}, \mathscr{H}_{\xi \zeta}^{(x)}, f_{v_{\xi}} g_{\mu_{\zeta}}\right\rangle-\left\langle f_{n_{\xi}} g_{m_{\xi}}, \mathscr{H}_{\xi \xi}^{(A)}, f_{v_{\zeta}} g_{\mu_{\zeta}}\right\rangle
$$

The quantity containing $\mathscr{H}_{\xi \xi}^{(A)}$ can be handled in exactly the same manner as $Z_{\xi \xi}^{A}$. The derivatives present in the double inner product involving $\mathscr{H}_{\xi 5}^{(x)}$ can be eliminated through integration by parts, resulting in the expressions 


$$
\begin{aligned}
& \left\langle f_{n_{\xi}} g_{m_{\xi}}, \frac{\partial^{2}}{\partial \xi^{2}} \frac{1}{\sqrt{\rho^{2}+\frac{t}{f(A)}}}{ }^{2} f_{v_{\xi}} g_{\mu_{\xi}}\right\rangle \\
& =\int_{\zeta m_{\xi}}^{\zeta m_{\xi}+1} \mathrm{~d} \zeta^{\prime} \int_{\zeta_{\mu_{\xi}}}^{\zeta_{\mu_{\xi}}+1} \mathrm{~d} \zeta \int_{-l_{\xi}}^{l_{\xi}} \mathrm{d} \xi \sin \left(k_{s}\left(l_{\xi}-|\xi|\right)\right) \\
& {\left[\frac{1}{\sqrt{\rho_{A}^{2}+\left(\frac{t}{f(A)}\right)^{2}}}+\frac{1}{\sqrt{\rho_{B}^{2}+\left(\frac{t}{f(A)}\right)^{2}}}-\frac{2 \cos \left(k_{s} l_{\xi}\right)}{\sqrt{\rho_{C}^{2}+\left(\frac{t}{f(A)}\right)^{2}}}\right]}
\end{aligned}
$$

and

$$
\begin{aligned}
& \left\langle f_{n_{\xi}} g_{m_{\xi}}, \frac{\partial^{2}}{\partial \xi \partial \zeta} \frac{1}{\sqrt{\rho^{2}+\left(\frac{t}{f(A)}\right)^{2}}}, f_{v_{\xi}} g_{\mu_{\xi}}\right\rangle \\
& =\int_{-l_{\xi}} \mathrm{d} \xi \int_{-l_{\zeta}}^{l_{\zeta}} \mathrm{d} \zeta^{\prime} \sin \left(k_{s}\left(l_{\xi}-|\xi|\right)\right) \sin \left(k_{s}\left(l_{\zeta}-\left|\zeta^{\prime}\right|\right)\right) \\
& {\left[\frac{1}{\sqrt{\rho_{D}^{2}+\left(\frac{t}{f(A)}\right)^{2}}}+\frac{1}{\sqrt{\rho_{E}^{2}+\left(\frac{t}{f(A)}\right)^{2}}}-\frac{1}{\sqrt{\rho_{F}^{2}+\left(\frac{t}{f(A)}\right)^{2}}}-\frac{1}{\sqrt{\rho_{\rho_{i}}^{2}+\left(\frac{t}{f(A)}\right)^{2}}}\right]}
\end{aligned}
$$

In equations (50) and (51), $\rho_{A-G}$ are functions of $\xi$ and $\zeta$ given by

$$
\begin{aligned}
& \rho_{A}^{2}=\left(\zeta-\zeta^{\prime}\right)^{2}+\left(\xi+\xi_{\nu_{\xi}}-\xi_{n_{\xi}}+l_{\xi}\right)^{2} \\
& \rho_{B}^{2}=\left(\zeta-\zeta^{\prime}\right)^{2}+\left(\xi+\xi_{\nu_{\xi}}-\xi_{n_{\xi}}-l_{\xi}\right)^{2} \\
& \rho_{C}^{2}=\left(\zeta-\zeta^{\prime}\right)^{2}+\left(\xi+\xi_{v_{\xi}}-\xi_{n_{\xi}}\right)^{2} \\
& \rho_{D}^{2}=\left(l_{\xi}-\zeta^{\prime}+\zeta_{\mu_{\xi}}-\zeta_{n_{\xi}}\right)^{2}+\left(\xi+\xi_{\nu_{\xi}}-\xi_{m_{\xi}}\right)^{2} \\
& \rho_{E}^{2}=\left(-\zeta^{\prime}+\zeta_{\mu_{\xi}}-\zeta_{n_{\zeta}}\right)^{2}+\left(\xi+\xi_{\nu_{\xi}}-\xi_{m_{\zeta}}+l_{\xi}\right)^{2} \\
& \rho_{F}^{2}=\left(-\zeta^{\prime}+\zeta_{\mu_{\xi}}-\zeta_{n_{\xi}}\right)^{2}+\left(\xi+\xi_{\nu_{\xi}}-\xi_{m_{\xi}}\right)^{2} \\
& \rho_{G}^{2}=\left(l_{\xi}-\zeta^{\prime}+\zeta_{\mu_{\xi}}-\zeta_{n_{\xi}}\right)^{2}+\left(\xi+\xi_{\nu_{\xi}}-\xi_{m_{\zeta}}+l_{\zeta}\right)^{2}
\end{aligned}
$$

\subsection{Numerical considerations}

2.2.1. Formation of the impedance matrix. As mentioned in the previous section, the discretization of the entire (M)MIC surface enclosing the microstrip discontinuity (Figure 2) has been performed. The reason for this approach is two-fold. On one hand, it allows the maximum utilization of symmetry inherently present in the open microstrip problem as will be discussed shortly. Secondly, when the discretization is performed in this manner, other circuit elements printed on the same substrate may be analysed without the re-evaluation of the impedance matrix elements. For the desired microstrip element, a simple routine correctly fills the impedance matrix from these pre-existing elements according to the discontinuity shape and the known boundary conditions. Therefore, if a design is to be made on a specific substrate (such as GaAs or alumina), impedance matrix elements can be pre-computed and stored in libraries, and re-used indefinitely for the synthesis of the desired performance. However, it must be noted that for very large problems, solving the matrix can be as time-consuming as generating the matrix elements.

The mesh of Figure 2 shows a total of $\left(N_{i} M_{i}\right),(i=x, y)$ node points resulting in a total of $\left(N_{x} M_{x}\right)^{2}+\left(N_{y} M_{y}\right)^{2}$ interactions for the direct terms $\left(Z_{x, x}, Z_{y y}\right)$ and $\left(N_{x} M_{x} N_{y} M_{y}\right)$ interactions for the cross-coupled terms $\left(Z_{y x}, Z_{x y}\right)$. Fortunately, this number can be reduced significantly by 
symmetry and reciprocity. From equations (21)-(25) it can be shown that the spatial dependence in the direct terms is an even function of the quantities $\left(x-x^{\prime}\right)$ and $\left(y-y^{\prime}\right)$. Furthermore, the cross-coupled interactions are odd functions of these quantities. Therefore, elements may be catalogued according to these properties, resulting in large reductions in computational effort. The number of elements computed for the particular submatrix $Z_{i j}$ is reduced to $N_{i} M_{j},(i, j=x, y)$ which is the square root of the previously given numbers. This is not true for shielded microstrip where the position of the cavity or waveguide wall is reflected in the spatial dependence of the Green's function. In this case, the interactions between subsections are not solely dependent on their relative position to each other, but also on their exact position in the cavity.

A three-dimensional view of the impedance matrix is shown in Figure 4. The matrix is toeplitz and diagonally dominant with the diagonal elements being the largest contribution by an order of magnitude. The large values of elements off the diagonal results from interactions of adjacent cells and their location in the impedance matrix depends on the ordering of the nodes.

2.2.2. Convergence with respect to the parameter $A$. The choice of the parameter $A$ in equation (26), influences both the accuracy and numerical convergence of the network parameters. The CPU time increase linearly with $A$ for the calculation of $Z_{\xi \zeta}^{A}$, while it is independent of $A$ in the computation of $Z_{\xi \zeta}^{\infty}$. Furthermore, the computer time for the computation of $Z_{\xi \zeta}^{A}$ is significantly greater than the time required for $Z_{\xi \zeta}^{\propto}$. Therefore, the value of $A$ must be chosen as small as possible, while still achieving numerical convergence. Figures 5 and 6 show the convergence of the phase of $S_{12}$ and the radiated power as a function of $A$. Table I shows the correspondence between the quantity $A_{r}$ defined in equation (26), and the parameter shown in the graph. As shown, the network parameters show no sensitivity to increasing $A_{t}$ above $0 \cdot 95$. Below $A_{t}=0 \cdot 9$, the accuracy of the phase of $S_{12}$ and the radiation loss gradually deteriorate until the estimated values become completely unacceptable at $A_{t}=0 \cdot 7$.

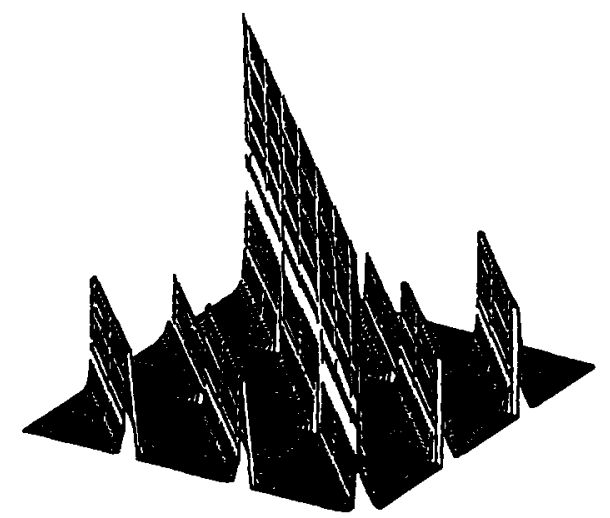

Figure 4. Impedance matrix

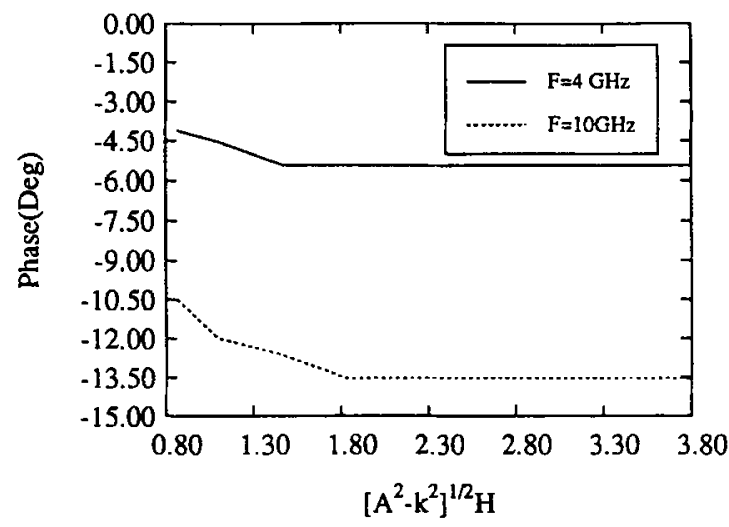

Figure 5. Convergence of the phase of $S_{12}$ as a function of the parameter $A$ for a microstrip corner discontinuity $\left(w=56 \mathrm{mil}(1.42 \mathrm{~mm}), h_{1}=56 \mathrm{mil}(1.42 \mathrm{~mm}), \epsilon_{r 1}=2\right)$ 


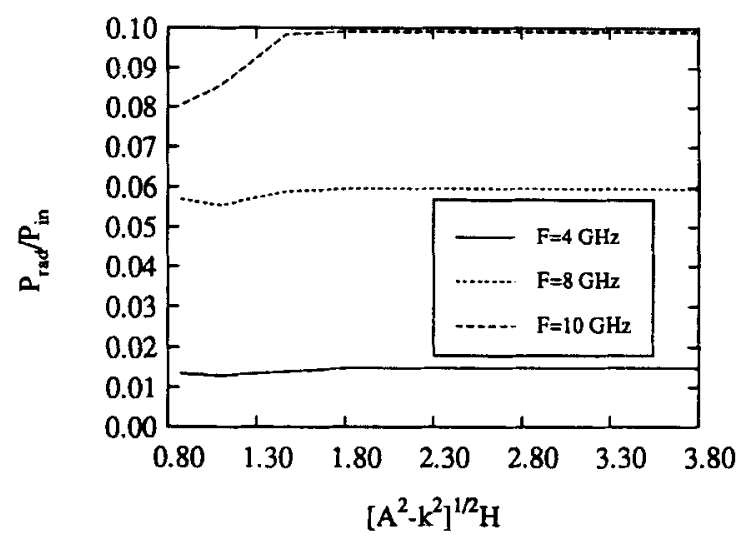

Figure 6. Convergence of the radiation loss as a function of the parameter $A$ for a microstrip corner discontinuity $\left(w=56 \mathrm{mil}(1.42 \mathrm{~mm}), h_{1}=56 \mathrm{mil}(1.42 \mathrm{~mm}), \mathbf{\epsilon}_{r 1}=2\right)$

Table I. Choice of $A$ microstrip corner discontinuity of Figures 5 and 6

\begin{tabular}{ccc}
\hline$\frac{A}{\lambda_{0}}(10 \mathrm{GHz})$ & $\sqrt{A^{2}-k^{2}} \cdot H$ & $\mathrm{~A}_{t}=\tanh \left(\sqrt{A^{2}-k^{2}} \cdot H\right)$ \\
\hline 20 & $0 \cdot 867$ & $0 \cdot 7$ \\
$24 \cdot 8$ & 1.098 & $0 \cdot 8$ \\
$32 \cdot 3$ & 1.472 & 0.9 \\
$39 \cdot 6$ & 1.832 & 0.95 \\
$56 \cdot 5$ & 2.647 & 0.99 \\
80.6 & 3.800 & 0.999 \\
\hline
\end{tabular}

\subsection{Computation of network parameters and radiated fields}

The solution of the matrix equation yields the current on the microstrip conducting strips as shown in Figure 7 for a corner discontinuity. This current clearly shows the formation of standing waves and the edge effect. In this simulation 500 basis functions were used, and the computer run required $870.8 \mathrm{CPU}$ seconds on an IBM RS 6000 . The matrix inversion used 6.7 per cent of the total run-time. From the current distribution the network parameters may be computed as shown in Reference 7 . The radiated fields may be obtained from the integral equation. This is done by applying a saddle-point integration technique as given in Reference 13.

\section{NUMERICAL RESULTS}

As discussed extensively in the literature, ${ }^{8}$ the efficiency of a printed antenna depends on the shape and size of the antenna and on the electric size and consistency of the dielectric substrate. An extensive study 8.9 has shown that the use of an appropriate combination of substrate and superstrate layers can improve the radiation performance by eliminating the surface waves. In monolithic array applications, where the individual antennas are fed by extensive feeding networks, an improvement in the radiation efficiency of the antennas by this technique will increase the parasitic radiation. As a result, the techniques for surface wave suppression have to be reevaluated.

In this paper, substrate/superstrate and two-layer substrate combinations made of duroid $\left(\epsilon_{r}=2 \cdot 2\right)$ and GaAs $\left(\epsilon_{r}=13\right)$, materials widely used in circuits, are considered and the effect of parasitic radiation is computed. Specifically, total radiation losses, and the percentages of surface wave and space wave power are evaluated as functions of the frequency and are compared to the single-layer substrate case.

- Substrate-superstrate configuration

Figure 8 shows the total radiated power as a function of frequency for a right-angle bend printed on a $40 \mathrm{mil}(1.02 \mathrm{~mm})$ duroid substrate with and without a 16 mil $(0.406 \mathrm{~mm}) \mathrm{GaAs}$ cover. 

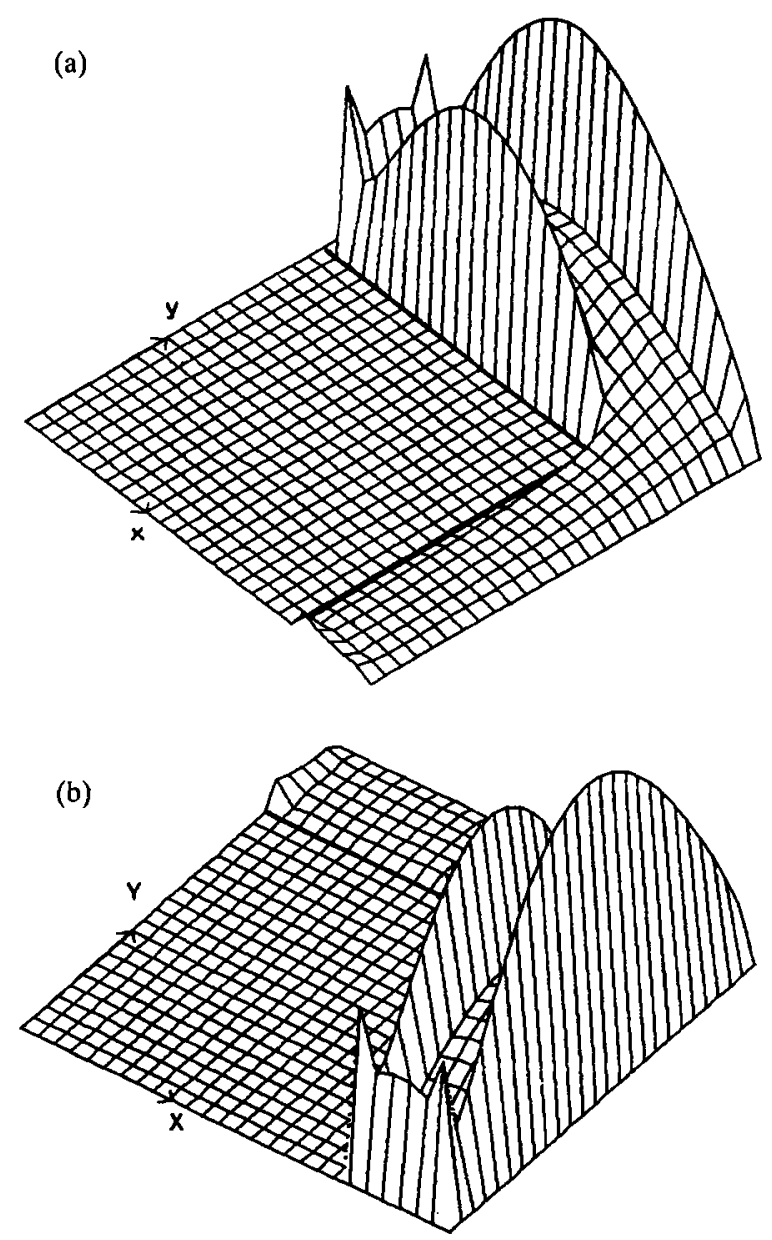

Figure 7. Current on microstrip corner excited by gap generators: $\epsilon_{r l}=2, h_{1}=56 \mathrm{mil}(1.42 \mathrm{~mm}), w=56 \mathrm{mil}(1.42 \mathrm{~mm})$ $\left(N_{x}+N_{y}=500,870 \cdot 8 \mathrm{CPU}\right.$ seconds). (a) $x$-directed current on corner; (b) $y$-directed current on corner

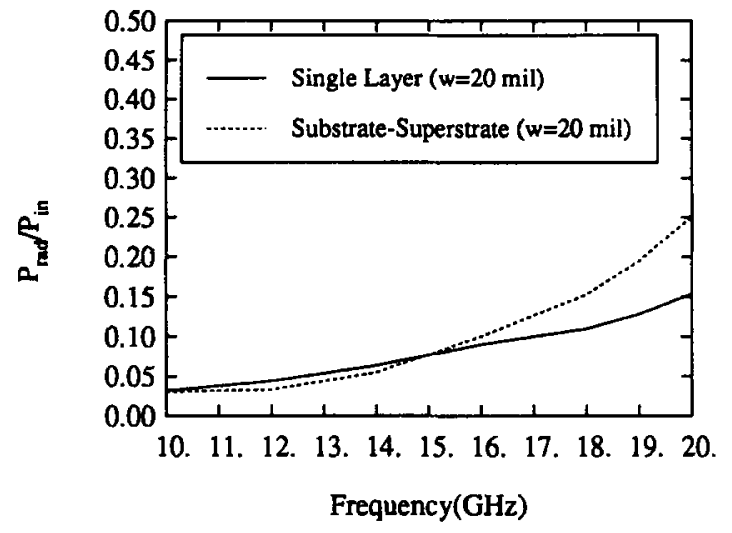

Figure 8. Total radiation loss for a microstrip corner discontinuity with a superstrate: $h_{1}=16$ mil $(0.406 \mathrm{~mm}), h_{2}=40$ mil $\left.(1.02 \mathrm{~mm}), \epsilon_{r 1}=13, \epsilon_{r 2}=2\right)$ and without a superstrate $\left(h_{1}=40 \mathrm{mil}(1.02 \mathrm{~mm}), \epsilon_{r 1}=2,\left(N_{x}+N_{y}=244,823.1 \mathrm{CPU}\right.\right.$ seconds/frequency)

The comparison shows clearly the effects of the superstrate from $10 \mathrm{GHz}$ to $20 \mathrm{GHz}$. In the lower half of the frequency band, the superstrate tends to reduce losses slightly. However, at higher frequencies, the total radiated power has increased by 67 per cent owing to the presence of the cover. As Figures 9 and 10 indicate, this excess radiated power comes from the enhancement of space wave radiation which is very desirable in antennas. In monolithic arrays printed on single layer dielectric substrate a careful design of the feeding network could provide parasitic radiation much lower than the primary radiation from the array. The replacement of the single layer by a substrate/superstrate configuration for array efficiency improvement could increase the power radiated by all the discontinuities included in the feeding structure substan- 


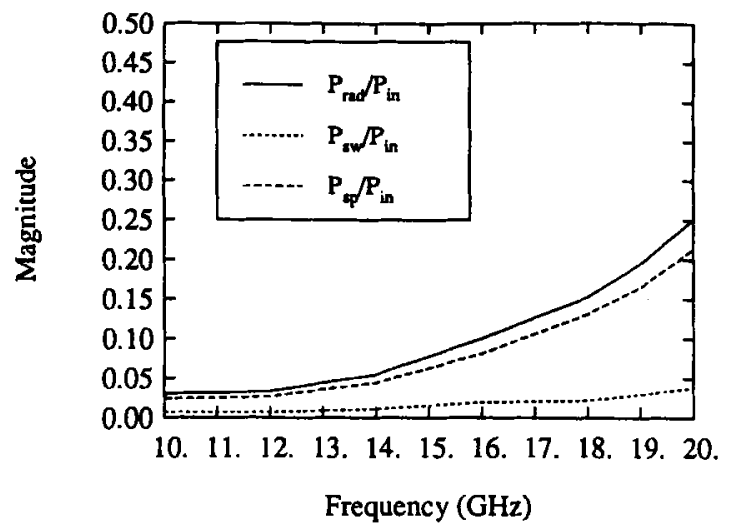

Figure 9. Radiation loss for a microstrip corner discontinuity with a superstrate $\left(h_{1}=16\right.$ mil $(0 \cdot 406 \mathrm{~mm}), h_{2}=40 \mathrm{mil}$ $\left.(1.02 \mathrm{~mm}), \epsilon_{r 1}=13, \epsilon_{r 2}=2\right)$

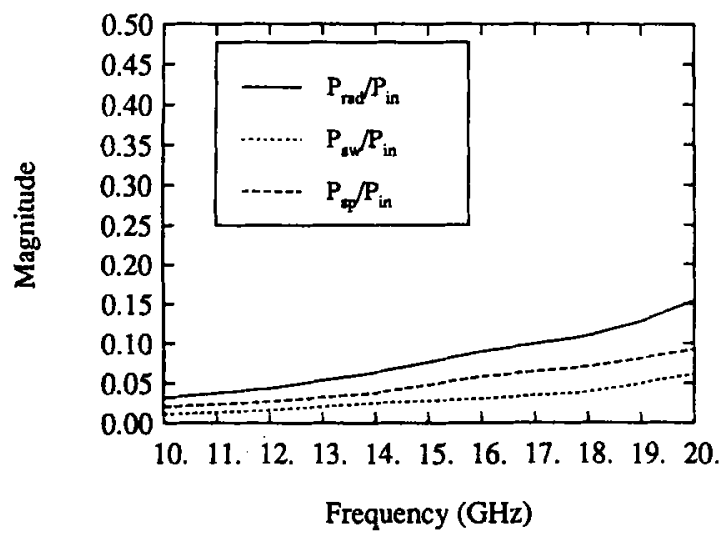

Figure 10. Radiation loss for a microstrip corner discontinuity on a single layer $\left(h_{1}=40 \mathrm{mil}(1.02 \mathrm{~mm}), \epsilon_{r 1}=2\right)$

tially. As a result, the level of the total parasitic radiation could become unacceptably high and could deteriorate the array pattern substantially.

- Two-layer substrate

In this case, two different comparisons are performed. At first the total power radiated by a right-angle bend printed on a 56 mil duroid is compared to the same bend printed on 40 mil duroid/16 mil GaAs substrate and shows a 20 per cent increase at the upper end of the frequency band mainly coming from the enhancement of the space wave radiation (see Figures 11 and 12). Much higher radiated power is observed when the geometry of the single-layer bend is modified to preserve the $100 \Omega$ input/output port characteristic impedance of the two-layer case. The excess loss in this case is due to the effects of electrically thick substrates which have been reported in Reference 1.

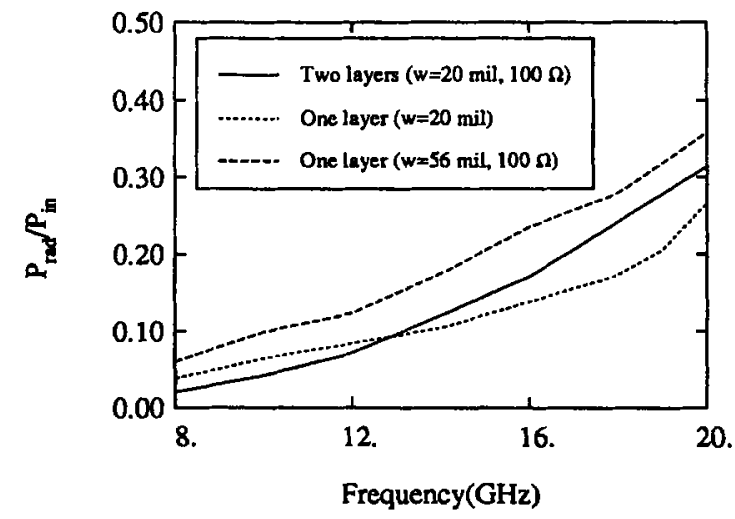

Figure 11. Total radiation loss for a microstrip corner discontinuity with a two-layer substrate $\left(h_{1}=16 \mathrm{mil}(0.406 \mathrm{~mm})\right.$, $\left.h_{2}=40 \mathrm{mil}(1.02 \mathrm{~mm}), \epsilon_{r 1}=13, \epsilon_{r 2}=2\right)$ and with a single layer substrate $\left(h_{1}=56 \mathrm{mil}(1.42 \mathrm{~mm}), \epsilon_{r 1}=2\right)$ 


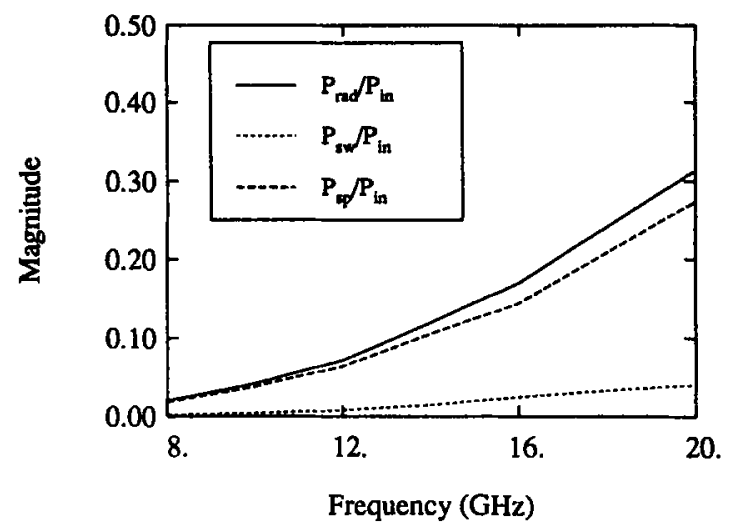

Figure 12. Radiation loss for a microstrip corner discontinuity with a two-layer substrate $\left(h_{1}=16 \mathrm{mil}(0.406 \mathrm{~mm})\right.$, $\left.h_{2}=40 \mathrm{mil}(1.02 \mathrm{~mm}), \epsilon_{r 1}=13, \epsilon_{r 2}=2\right)$

In both of the above cases, the frequency range was chosen so that only one mode is excited in the substrate. Furthermore, the presence of the superstrate or of a second layer with a higher dielectric constant tends to reduce the power of the excited surface wave and increase the power radiated into space waves. These effects have to be taken into account when techniques for enhancement of the radiation efficiency are applied in arrays fed by extensive feeding networks.

\section{CONCLUSION}

Radiation losses for microstrip corner discontinuities printed on substrates with one and two dielectric layers, and/or a superstrate are presented. The losses were evaluated with a spacedomain integral equation approach and were separated into space and surface wave components. It was found that a superstrate, often used for protection or gain enhancement of antenna elements, may increase the loss of the microstrip feed network considerably. This would result in lower overall gain. Therefore, a tradeoff exists between the enhancement of radiation from the antennas and the undesirable radiation in the feed network.

Another comparison between a corner discontinuity on a single layer of duroid, and on a twolayer structure (GaAs/duroid) having the same total thickness, showed that the radiation losses were comparable when the conducting strips had the same width. However, it was found that the loss was influenced by the strip width. Specifically, when the width in the single-layer case was increased to create the same characteristic impedance as the two-layer case, the loss was substantially higher.

\section{APPENDIX A}

This appendix contains the functions included in the expression of the Green's function in equations (3)-(4). The functions are given below for three different substrate configurations.

\section{- Substrate-superstrate configuration}

The functions for the substrate-superstrate geometry of Figure 1(a) in the region $\left(0<Z<h_{1}\right)$ are given by

$$
\begin{aligned}
\mathcal{N}_{\xi \xi}(\lambda) & =\lambda \\
\mathcal{N}_{z \xi}(\lambda) & =\frac{j}{u_{1}} \\
\mathscr{Z}_{\xi \xi} & =u_{1} \cosh \left[j u_{1}\left(z-h_{1}\right)\right]-u_{0} \sinh \left[j u_{1}\left(z-h_{1}\right)\right] \\
\mathscr{Z}_{z \xi} & =\left[u_{1}^{2} u_{2} f_{1}(\lambda)-u_{0}^{3} f_{2}(\lambda)\right] \cosh \left[j u_{1}\left(z-h_{1}\right)\right] \\
& +u_{1} u_{0}\left[u_{0} f_{2}(\lambda)-\epsilon_{r 1} u_{2} f_{1}(\lambda)\right] \sinh \left[j u_{1}\left(z-h_{1}\right)\right]
\end{aligned}
$$


with $u_{i}=\sqrt{ } k_{i}^{2}-\lambda^{2}$. The expressions $f_{1}(\lambda)$ and $f_{2}(\lambda)$ are the characteristic equations for surface wave modes given by

$$
\begin{aligned}
f_{1}(\lambda) & =u_{0}\left[u_{1} \cosh \left(j u_{1} h_{1}\right)+u_{2} \operatorname{coth}\left(j u_{2} h_{2}\right) \sinh \left(j u_{1} h_{1}\right)\right] \\
& +u_{1}\left[u_{1} \sinh \left(j u_{1} h_{1}\right)+u_{2} \operatorname{coth}\left(j u_{2} h_{2}\right) \cosh \left(j u_{1} h_{1}\right)\right] \\
f_{2}(\lambda) & =u_{1}\left[\epsilon_{r 2} u_{1} \sinh \left(j u_{1} h_{1}\right) \operatorname{coth}\left(j u_{2} h_{2}\right)+\epsilon_{r 1} u_{2} \cosh \left(j u_{1} h_{1}\right)\right] \\
& +\epsilon_{r 1} u_{0}\left[\epsilon_{r 2} u_{1} \operatorname{coth}\left(j u_{2} h_{2}\right) \cosh \left(j u_{1} h_{1}\right)+\epsilon_{r 1} u_{2} \sinh \left(j u_{1} h_{1}\right)\right]
\end{aligned}
$$

- Two-layer substrate

The functions for the two-layer case in Figure 1(b) for the region $(Z>0)$ are given by

$$
\begin{gathered}
\mathcal{N}_{\xi \xi}(\lambda)=\left[u_{1} \cosh \left(j u_{1} h_{1}\right)+u_{2} \sinh \left(j u_{1} h_{1}\right) \operatorname{coth}\left(j u_{2} h_{2}\right)\right] \lambda \\
\mathcal{N}_{z \xi}(\lambda)=j \epsilon_{r 1} u_{0}^{2}\left[\epsilon_{r 2} u_{1} \cosh \left(j u_{1} h_{1}\right) \operatorname{coth}\left(j u_{2} h_{2}\right)+\epsilon_{r 1} u_{2} \sinh \left(j u_{1} h_{1}\right)\right] \\
{\left[u_{1} \cosh \left(j u_{1} h_{1}\right)+u_{2} \operatorname{coth}\left(u_{2} h_{2}\right) \sinh \left(j u_{1} h_{1}\right)\right]} \\
-j u_{1}^{2}\left[u_{1} \sinh \left(j u_{1} h_{1}\right)+u_{2} \operatorname{coth}\left(j u_{2} h_{2}\right) \cosh \left(j u_{1} h_{1}\right)\right] \\
{\left[\epsilon_{r 2} u_{1} \operatorname{coth}\left(j u_{2} h_{2}\right) \sinh \left(j u_{1} h_{1}\right)+\epsilon_{r 1} u_{2} \cosh \left(j u_{1} h_{1}\right)\right]} \\
\mathscr{W}_{\xi \xi}=\mathscr{W}_{z \xi}=\mathrm{e}^{-j u_{1} z^{z}}
\end{gathered}
$$

- Single-layer substrate

The expressions for the single-layer case may be obtained from equations (A5)-(A8). After some simplification they can be written as

$$
\begin{aligned}
\mathcal{N}_{\xi \xi}(\lambda) & =\sinh \left(j u_{1} h_{1}\right) \lambda \\
\mathcal{N}_{z \xi}(\lambda) & =j\left[\left(1-\epsilon_{r}\right) \sinh j u_{1} h \cosh j u_{1} h\right] \lambda^{2} \\
f_{1}(\lambda) & =u_{0} \sinh j u_{1} h+u_{1} \cosh j u_{1} h \\
f_{2}(\lambda) & =\epsilon_{r} u_{0} \cosh j u_{1} h+u_{1} \sinh j u_{1} h
\end{aligned}
$$

\section{APPENDIX B}

The quadruple integrals (25) for direct coupled $x-x$ interactions can be written

$$
\begin{aligned}
& \left\langle f_{n_{x}}\left(x^{\prime}\right) g_{m_{x}}\left(y^{\prime}\right), J_{0}(\lambda \rho), f_{v_{x}}(x) g_{\mu_{x}}(y)\right\rangle \\
& =\iint_{S^{\prime}} \mathrm{d} x^{\prime} \mathrm{d} y^{\prime} \iint_{S} \mathrm{~d} x \mathrm{~d} y\left[f_{n_{x}}\left(x^{\prime}\right) g_{m_{.}}\left(y^{\prime}\right) J_{0}(\lambda \rho) f_{v_{x}}(x) g_{\mu_{x}}(y)\right] \\
& \left\langle f_{n_{x}}\left(x^{\prime}\right) g_{m_{x}}\left(y^{\prime}\right), \frac{\partial^{2}}{\partial x^{2}} J_{0}(\lambda \rho), f_{\nu_{x}}(x) g_{\mu_{x}}(y)\right\rangle \\
& =\iint_{S^{\prime}} \mathrm{d} x^{\prime} \mathrm{d} y^{\prime} \iint_{S} \mathrm{~d} x \mathrm{~d} y\left[f_{n_{x}}\left(x^{\prime}\right) g_{m_{x}}\left(y^{\prime}\right) \frac{\partial^{2}}{\partial x^{2}} J_{0}(\lambda \rho) f_{v_{x}}(x) g_{\mu_{x}}(y)\right]
\end{aligned}
$$

and for cross coupled $x-y$ terms

$$
\begin{aligned}
& \left\langle f_{n_{x}}\left(x^{\prime}\right) g_{m_{x}}\left(y^{\prime}\right), \frac{\partial^{2}}{\partial x \partial y} J_{0}(\lambda \rho), f_{v_{y}}(x) g_{\mu_{y}}(y)\right\rangle \\
= & \iint_{S^{\prime}} \mathrm{d} x^{\prime} \mathrm{d} y^{\prime} \iint_{S} \mathrm{~d} x \mathrm{~d} y\left[f_{n_{x}}\left(x^{\prime}\right) g_{m_{x}}\left(y^{\prime}\right) \frac{\partial^{2}}{\partial x \partial y} J_{0}(\lambda \rho) f_{v_{y}}(y) g_{\mu_{y}}(x)\right]
\end{aligned}
$$

Employing the integral representation for the first-order Bessel function in equations (31)-(32), the above may be simplified to the expressions 


$$
\begin{aligned}
& \left\langle f_{n_{x}}\left(x^{\prime}\right) g_{m_{x}}\left(y^{\prime}\right), J_{0}(\lambda \rho), f_{v_{x}}(x) g_{\mu_{x}}(y)\right\rangle \\
& =\frac{1}{2 \pi} \int_{-\pi}^{\pi} \mathrm{e}^{j \lambda\left(x_{v_{x}}-x^{\prime} n_{x}\right) \cos (\phi)} \mathrm{e}^{j \lambda\left(y_{\mu_{1}}-y^{\prime} m_{x}\right) \sin (\phi)} \\
& (\Re(\lambda \cos (\phi)))^{2} u(\lambda \sin (\phi)) \cup(-\lambda \sin (\phi)) \mathrm{d} \phi \\
& \left\langle f_{n_{x}}\left(x^{\prime}\right) g_{m_{x}}\left(y^{\prime}\right), \frac{\partial^{2}}{\partial x^{2}} J_{0}(\lambda \rho), f_{\nu_{x}}(x) g_{\mu_{x}}(y)\right\rangle \\
& =\frac{1}{2 \pi} \int_{-\pi}^{\pi}(j \lambda \cos \phi)^{2} e^{j \lambda\left(x_{\nu_{x}}-x^{\prime}{ }_{n_{x}}\right) \cos (\phi)} e^{j \lambda\left(v_{\mu_{x}}-y_{m_{*}^{\prime}}{ }_{m_{k}}\right) \sin (\phi)} \\
& \mathscr{R}(\lambda \cos (\phi))^{2} u(\lambda \sin (\phi)) u(-\lambda \sin (\phi)) d \phi \\
& \left\langle f_{n_{x}}\left(x^{\prime}\right) g_{m_{x}}\left(y^{\prime}\right), \frac{\partial^{2}}{\partial x \partial y} J_{0}(\lambda \rho), f_{v_{y}}(y) g_{\mu_{y}}(x)\right\rangle \\
& =\frac{1}{2 \pi} \int_{-\pi}^{\pi}(j \lambda \sin \phi)(\mathrm{j} \lambda \cos \phi) \mathrm{e}^{j \lambda\left(x_{\mu_{y}}-x^{\prime}{ }_{{ }_{x}}\right) \cos (\phi)} \mathrm{e}^{j \lambda\left(y_{v_{y}}-y^{\prime}{ }_{m_{y}}\right) \sin (\phi)} \\
& \mathscr{R}(\lambda \cos (\phi)) \mathscr{R}(\lambda \sin (\phi)) \mathcal{u}(\lambda \cos (\phi)) \cup(-\lambda \sin (\phi)) \mathrm{d} \phi
\end{aligned}
$$

The quantities $\mathscr{R}$ and $\mathcal{U}$ are convergent series having the form

$$
\begin{aligned}
& \mathscr{R}(\lambda \cos (\phi))=\sum_{k=0}^{\infty} \mathrm{A}_{2 k}(\lambda \cos (\phi))^{2 k} \\
& \mathcal{U}(\lambda \cos (\phi))=\sum_{l=0}^{\infty} B_{l}(\lambda \cos (\phi))^{l}
\end{aligned}
$$

where

$$
\begin{aligned}
A_{2 k} & =\frac{2}{k^{2 k+1}}\left(\left[1-\cos \left(k l_{x}\right)\right]+\sum_{\mu=1}^{k}(-1)^{\mu} \frac{\left(k l_{x}\right)^{2 \mu}}{(2 \mu)^{2}}\right) \\
B_{2 n} & =(-1)^{n}\left(l_{x}\right)^{2 n} \\
B_{2 n+1} & =j(-1)^{n}\left(l_{x}\right)^{2 n+1}
\end{aligned}
$$

considering the integral representation for the zeroth-order Bessel function these expressions can be re-written as a summation of derivatives of the zeroth-order Bessel function appearing in the main text (equations (33)-(35)).

\section{REFERENCES}

1. N. G. Alexopoulos, P. B. Katehi and D. Rutledge, 'Substrate optimization for integrated circuit applications', IEEE Trans. Microwave Theorv Tech., MTT-31, 550-557 (1983).

2. P. B. Katehi and N. G. Alexopoulos, 'Frequency-dependent characteristics of microstrip discontinuities in millimeterwave integrated circuits', IEEE Trans. Microwave Theory Tech., MTT-33, 1029-1035 (1985).

3. J. R. Mosig, 'Arbitrarily shaped microstrip structures and their analysis with a mixed potential integral equation', IEEE Trans. Microwave Theory Tech., MTT-36, 314-323 (1988).

4. R. W. Jackson and D. M. Pozar, 'Full-wave analysis of microstrip open-end and gap discontinuities', IEEE Trans. Microwave Theory Tech., MTT-33, 1036-1042 (1985).

5. R. Jackson, 'Full-wave finite element analysis of irregular microstrip discontinuities', IEEE Trans. Microwave Theory Tech., MTT-37, 81-89 (1989)

6. W. P. Harokopus Jr. and P. B. Katehi, 'An accurate characterization of open microstrip discontinuities including radiation losses', IEEE Microwave Symposium Digest, June 1989, pp. 231-234.

7. W. P. Harokopus Jr. and P. B. Katehi, 'Characterization of open microstrip discontinuities on multilayer substrates including radiation losses", IEEE Trans. Microwave Theory Tech., MTT-37, 1964-1972 (1989).

8. N. G. Alexopoulos and D. R. Jackson, 'Fundamental superstrate (cover) effects on printed circuit antennas', IEEE Transactions on Antennas and Propagation, AP-32, 807-814 (1984).

9. N. G. Alexopoulos, D. R. Jackson and P. B. Katehi, 'Criteria for nearly omnidirectional patterns for printed antennas, IEEE Transactions on Antennas and Propagation, AP-33, 195-205 (1985). 
10. W. P. Harokopus Jr. and P. B. Katehi, 'Radiation properties of microstrip discontinuities', IEEE Ap Digest, June 1989 , pp. $1703-1706$.

11. W. P. Harokopus Ir., P. B. Katehi, W. Ali-Ahmad and G. M. Rebeiz, 'Surface wave excitation in antenna feed networks', 1990 Radio Science Meeting Program and Abstracts, May 1990, p. 337.

12. T. S. Horng, S. C. Wu, H. Y. Yang and N. G. Alexopoulos, 'A generalized method for the distinction of radiation and surface wave losses in microstrip discontinuities', IEEE Microwave Symposium Digest, May 1990, pp. 1055-1058.

13. W. P. Harokopus Jr. and P. B. Katehi. 'Surface wave excitation from open microstrip discontinuities', Accepted for publication in IEEE Trans. Microwave Theory Tech.

14. A. Sommerfeld, Partial Differential Equations in Physics, Academic Press, New York, 1949.

15. R. F. Harrington, Field Computation By Moment Methods, Macmillan, New York, 1968

16. J. C. Rautio and R. F. Harrington, 'An electromagnetic timeharmonic analysis of shielded microstrip circuits', IEEE Trans. Microwave Theory Tech., MTT-35, 726-730 (1987).

17. P. B. Katehi and N. G. Alexopoulos, 'Real axis integration of Sommerfeld integrals with applications to printed circuit antennas', J. Math. Phys., 24, 253-260 (1983).

\section{Authors' biographies:}

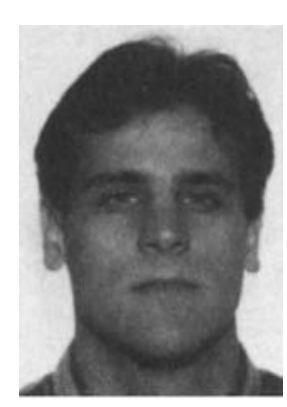

William P. Harokopus was born in Detroit, MI, in 1963. He received his BS (1985) and MS (1986) degrees in electrical engineering from the University of Michigan, Ann Arbor. He is currently pursuing his Ph.D. at Michigan where he works in the Radiation Lab as a graduate research assistant. His dissertation will be on the high frequency characterization of open microstrip discontinuities.

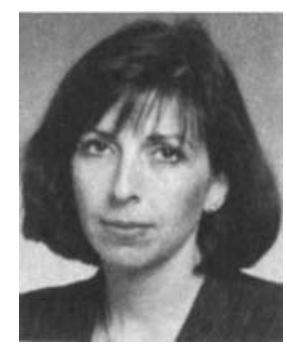

Linda P. B. Katehi received the BSEE degree from the National Technical University of Athens, Greece, in 1977 and the MSEE and Ph.D. degrees from the University of California, Los Angeles, in 1981 and 1984 respectively. In September 1984 she joined the faculty of the EECS Department of the University of Michigan, Ann Arbor. Since then, she has been involved in the modelling and computer-aided design of millimetreand near-millimetre-wave monolithic circuits and antennas. In 1984 she received the $\mathrm{W}$. P. King Award and in 1985 the S. A. Schelkunoff Award from the Antennas and Propagation Society. In 1987 she received an NSF Presidential Young Investigator Award and an URSI Young Scientist Fellowship. She is a member of IEEE AP-S, MTT-S and Sigma Xi. 